\title{
Outcomes of surgery for infective endocarditis in children: A 30-year experience
}

\author{
Brandon Khoo, MD, ${ }^{a}$ Edward Buratto, MBBS, ${ }^{\text {a,b,c }}$ Tyson A. Fricke, MBBS, BMedSci, ${ }^{\text {a,b,c }}$ \\ Ben Gelbart, MBBS, FRACP, FCICM, ${ }^{\mathrm{b}, \mathrm{c}, \mathrm{d}}$ Christian P. Brizard, MD, MS, ${ }^{\mathrm{a}, \mathrm{b}, \mathrm{c}}$ \\ Johann Brink, MBChB, FRACS, ${ }^{\text {a,b,c }}$ Yves d'Udekem, MD, PhD, FRACS, ${ }^{a, b, c}$ and \\ Igor E. Konstantinov, MD, PhD, FRACS ${ }^{\mathrm{a}, \mathrm{b}, \mathrm{c}, \mathrm{e}}$
}

\section{ABSTRACT}

Background: Infective endocarditis (IE) is rare in children. Limited data have been reported on long-term outcomes of children who undergo surgery for IE.

Methods: Data were retrospectively obtained from medical records for all children who underwent surgery for IE.

Results: Between 1987 and 2017, 138 children with IE required surgery (mean age, $8.3 \pm 6.5$ years). The majority of children ( $80.4 \%$ [111 out of 138]) had underlying cardiac structural anomalies. Prior heart surgery was performed in $50.7 \%$ of patients ( 70 out of 138 ), including $19.6 \%$ (27 out of 138 ) who had valve replacement. Operative mortality was $5.8 \%$ (8 out of 138). Mean follow-up time was $9.7 \pm 7.6$ years. Long-term survival at 5 and 25 years was $91.5 \%(95 \%$ confidence interval, $85.1 \%-95.2 \%)$ and $79.1 \%$ (95\% confidence interval, $66.3 \%-87.5 \%$ ), respectively. Risk factors associated with death were: age (hazard ratio $[\mathrm{HR}], 0.88 ; P=.015)$, prosthetic valve $\mathrm{IE}(\mathrm{HR}, 3.86 ; P=.02)$, coagulase-negative staphylococci (HR, 4.52; $P=.015)$, increased duration of preoperative antibiotic therapy (HR, 1.02; $P=.009$ ), shock (HR, 3.68; $P=.028)$, and aortic valve replacement (HR, 3.22; $P=.044)$. In patients with left-sided IE, risk factors independently associated with death were heart failure (HR, 18.8; $P=.025)$ and vegetation size adjusted to body surface area (HR, 1.06; $P=.008)$. Freedom from recurrent endocarditis was $94.7 \%$ (95\% confidence interval, $87.7 \%-97.8 \%$ ) at 25 years.

Conclusions: Children undergoing surgery for IE had good long-term survival and recurrence of IE was uncommon. Surgery during the active phase of endocarditis did not increase risk of mortality or reoperation. In patients with left-sided IE, vegetation size adjusted for patient body surface area was identified as a risk factor for death, and a useful indicator of prognosis. ( $\mathrm{J}$ Thorac Cardiovasc Surg 2019;158:1399-409)

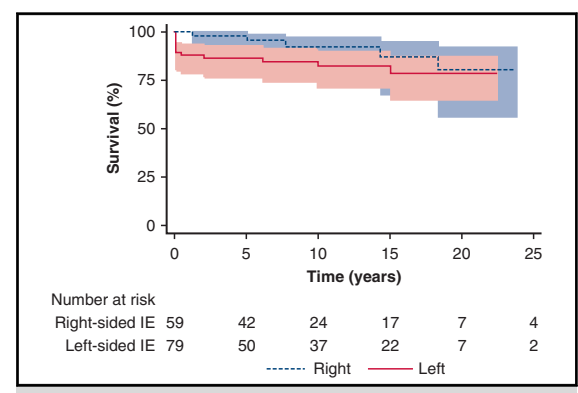

Survival for left-sided and right-sided infective endocarditis.

\section{Central Message}

Children undergoing surgery for IE have good survival and a low recurrence of IE. In patients with left-sided IE, vegetation size adjusted for patient body surface area is a risk factor for death.

\section{Perspective}

Long-term data on the outcomes of surgery for IE in children is limited. This study describes outcomes of surgery for IE in the largest cohort of pediatric patients ever reported.

See Commentaries on pages 1410 and 1411.
Infective endocarditis (IE) accounts for 0.1 to 0.8 per 1000 pediatric admissions. ${ }^{1}$ Recent studies have suggested that the incidence of $\mathrm{IE}$ in children is increasing due to improving survival of patients with corrected congenital heart disease (CHD). ${ }^{2}$ Additionally, an increasing number of children without underlying cardiac defects are

\footnotetext{
From the Departments of a Cardiac Surgery and ${ }^{\mathrm{d}}$ Intensive Care, The Royal Children's Hospital, Melbourne, Australia; 'bepartment of Pediatrics, The University of Melbourne, Melbourne, Australia; ' Murdoch Children's Research Institute, Melbourne, Australia; and 'Melbourne Children's Centre for Cardiovascular Genomics and Regenerative Medicine, Melbourne, Australia.

Supported by the Victoria Government's Operational Infrastructure Support Program. Dr Buratto is a recipient of a Reg Worcester Scholarship from the Royal Australasian College of Surgeons. Dr Fricke is a recipient of a Postgraduate Scholarship from the National Health and Medical Research Council (1134203). Dr d'Udekem is a National Health and Medical Research Council Clinician Practitioner Fellow (1082186).
}

Received for publication Nov 27, 2018; revisions received May 26, 2019; accepted for publication June 10, 2019; available ahead of print Aug 2, 2019.

Address for reprints: Igor E. Konstantinov, MD, PhD, FRACS, Department of Cardiac Surgery, Royal Children's Hospital, Flemington Rd, Parkville, 3029, Australia (E-mail: igor.konstantinov@ rch.org.au).

0022-5223/\$36.00

Crown Copyright $@ 2019$ Published by Elsevier Inc. on behalf of The American Association for Thoracic Surgery

https://doi.org/10.1016/j.jtcvs.2019.06.024 


\section{Abbreviations and Acronyms \\ $\mathrm{CHD}=$ congenital heart disease \\ CoNS $=$ coagulase-negative staphylococci \\ IE $=$ infective endocarditis}

developing IE secondary to the use of central lines and other invasive procedures in neonatal and pediatric intensive care settings.

Currently, pediatric recommendations for the management of IE are extrapolated from the guidelines developed for adults. A majority of children with IE are managed medically with antibiotic therapy and supportive care. ${ }^{3}$ Cardiac surgery is performed in approximately $20 \%$ of pediatric admissions for IE. ${ }^{2}$ Surgery is usually reserved for children, who are at increased risk of stroke, hemodynamic compromise, or failure of antibiotic therapy. ${ }^{4}$

Long-term data on the outcomes of surgery for IE in children is lacking. The aim of this study was to determine the outcomes of children who underwent surgery for IE at a single large-volume institution.

\section{METHODS \\ Patients}

All patients who underwent surgery for IE at the Royal Children's Hospital, Melbourne, Australia, from January 1, 1987, to January 1, 2017, were included in this study. Ethics approval was obtained from the Royal Children's Hospital Human Research Ethics Committee (No. 36250A).

Patients were identified using the modified Duke Criteria for possible or definite IE, accounting for microbiologic, echocardiographic, and clinical criteria. ${ }^{5}$ Patients were also included if clear evidence of IE was found intraoperatively.

Data were obtained from retrospective chart review. Follow-up data were collected by correspondence with the general practitioners and cardiologists. Follow-up was complete if patient contact last occurred within 2 years of the end of the study period. Operative mortality was defined as death within 30 days of surgery or before hospital discharge. Early reoperation was defined as surgical reintervention occurring within 30 days of surgery or before hospital discharge. Patients were classified as having cardiogenic shock if they required parenteral inotropic/vasopressor agents or mechanical support in conjunction with documented clinical evidence of shock. Patients were considered to have active IE if they were receiving intravenous antibiotics until the time of surgery. Patients who had completed antibiotic treatment before surgery were classified as having treated IE. ${ }^{6}$ The date of diagnosis of IE was recorded as the time point at which the patient met Duke Criteria for IE, otherwise, the start date of intravenous antibiotics or date of cardiac surgery was used.

\section{Statistical Analysis}

All data were analyzed using STATA version 14 (Stata Corp, College Station, Tex). Unless otherwise specified, all continuous data are expressed as mean \pm standard deviation. Categorical data were compared between groups using the $\chi^{2}$ test or the Fisher exact test if group size $<10$. Continuous data were compared between groups using the Kruskal-Wallis test.

Time dependent end points, such as survival and freedom from reoperation, were analyzed using the Kaplan-Meier method, with time commencing at the date of surgery for IE.
Risk factors evaluated using univariable Cox regression were age at operation, pre-existing $\mathrm{CHD}$, history of cardiac surgery, prior IE, active infection, catheter-associated IE, left-sided IE, prosthetic valve IE, Staphylococcus aureus infection, coagulase-negative staphylococci (CoNS) infection, time from diagnosis to surgery, time from vegetation detection to surgery, time from first embolic event to surgery, length of preoperative antibiotic therapy, severe valvular insufficiency, embolic events, heart failure, shock, preoperative intensive care unit admission, perivalvular abscess, vegetation size to body surface area ratio, and replacement of individual valves at surgery.

Variables with at least moderate evidence against the null hypothesis $(P<.10)$ were included in a multivariable model. Explanatory variables were tested for collinearity using the $\chi^{2}$ test, $t$ test, variance inflation factor, or Pearson correlation coefficient as appropriate. The weaker predictor from a pair of strongly correlated variables was excluded from the multivariable model. The model was then refined using the backward elimination method.

\section{RESULTS}

A total of 138 patients underwent surgery for 138 episodes of IE from January 1, 1987, to January 1, 2017. Mean follow-up time was $9.7 \pm 7.6$ years (median, 8.5 years; interquartile range [IQR], 3.3-16.4 years), $96.4 \%$ of patients (133 out of 138) had follow-up, $88.4 \%$ of patients (122 out of 138 ) had been reviewed within 12 months or had at least 5 years of follow-up. Baseline demographic characteristics of our cohort are in Table 1 . The median age at operation was 8 years (mean, $8.3 \pm 6.5$ years; IQR, 1.3-14.0 years). Infants aged $<1$ year constituted $20.3 \%$ of all IE patients (28 out of 138).

\section{Cardiac History}

Underlying CHD was present in $77.5 \%$ of patients (107 out of 138) (Table 1). Ventricular septal defect was the most common lesion, found in $32.6 \%$ of patients (45 out of 138), followed by bicuspid aortic valve (17 out of $138[12.3 \%])$ and aortic stenosis (17 out of 138 $[12.3 \%])$.

In $50.7 \%$ of patients (70 out of 138), there was a history of at least 1 previous cardiac operation. Of these patients, $61.4 \%$ (43 out of 70 ) had previous valve surgery, $62.8 \%$ of whom (27 out of 43 ) had a total of 34 prosthetic valves: $82.4 \%$ of these prostheses ( 28 out of 34 ) had signs of IE. The mean time between the final operation and subsequent surgery for IE was $3.8 \pm 4.4$ years (median, 1.8 years; IQR, 0.2-6.3 years). Intracardiac synthetic material (other than suture) was present in $38.4 \%$ of patients (53 out of 138) before diagnosis of IE.

\section{Location of IE}

Patients with biventricular circulation had isolated left-sided IE (60 out of 138 [43.5\%]), isolated right-side IE (59 out of 138 [42.7\%]) or IE affecting both sides of the heart (9 out of $138[6.5 \%]$ ). The remaining patients (10 out of 138 [7.2\%]) had IE in a univentricular 
TABLE 1. Demographic characteristics of study patients $(\mathbf{N}=\mathbf{1 3 8})$

\begin{tabular}{|c|c|}
\hline Characteristic & Result \\
\hline \multicolumn{2}{|l|}{ Sex } \\
\hline Male & $66(47.8)$ \\
\hline Female & $72(52.2)$ \\
\hline Age $(y)$ & $7.9(1.3-14.0)$ \\
\hline$<1$ & $28(20.3)$ \\
\hline$\geq 1$ & $111(82.2)$ \\
\hline Height (cm) & $124(77-159)$ \\
\hline Weight (kg) & $27.0(9.5-49.7)$ \\
\hline Indigenous Australian & $11(8.0)$ \\
\hline Prior cardiac operation & $70(50.7)$ \\
\hline Prior valve surgery & $43(31.2)$ \\
\hline Prior valve replacement & 27 (19.6) \\
\hline Prior episode of infective endocarditis & $4(2.9)$ \\
\hline Pre-existing congenital heart disease & $107(77.5)$ \\
\hline Prior rheumatic heart disease & $4(2.9)$ \\
\hline \multicolumn{2}{|l|}{ Cardiac anomalies } \\
\hline Ventricular septal defect & $45(32.6)$ \\
\hline Atrial septal defect/patent foramen ovale & $20(14.5)$ \\
\hline Bicuspid aortic valve & $17(12.3)$ \\
\hline Aortic stenosis & $17(12.3)$ \\
\hline Pulmonary atresia & $14(10.1)$ \\
\hline Coarctation of aorta & $13(9.4)$ \\
\hline Patent ductus arteriosus & $12(8.7)$ \\
\hline Transposition of great arteries & $9(6.5)$ \\
\hline Tetralogy of Fallot & $7(5.1)$ \\
\hline Hypoplastic left heart syndrome & $6(4.3)$ \\
\hline Truncus arteriosus & $5(3.6)$ \\
\hline Atrioventricular septal defect & $5(3.6)$ \\
\hline Major aortopulmonary collateral arteries & $5(3.6)$ \\
\hline Pulmonary stenosis & $4(2.9)$ \\
\hline Double outlet right ventricle & $3(2.2)$ \\
\hline Ebstein's anomaly & $3(2.2)$ \\
\hline Hypoplastic right heart syndrome & $3(2.2)$ \\
\hline Dextrocardia & $1(0.7)$ \\
\hline Mitral stenosis & $1(0.7)$ \\
\hline Tricuspid atresia & $1(0.7)$ \\
\hline Double inlet left ventricle & $1(0.7)$ \\
\hline Total anomalous pulmonary venous drainage & $1(0.7)$ \\
\hline \multicolumn{2}{|l|}{ Valve involved } \\
\hline Aortic & $39(28.3)$ \\
\hline Mitral & $37(26.8)$ \\
\hline Tricuspid & $24(17.4)$ \\
\hline Pulmonary/conduit & $39(28.3)$ \\
\hline
\end{tabular}

Values are presented as $\mathrm{n}(\%)$ or median (interquartile range).

circulation. The outcomes of the patients with involvement of systemic circulation were analyzed together. Native valve IE (90 out of 138 [65.2\%]) accounted for the majority of cases; $20.3 \%$ of patients (28 out of 138 ) had prosthetic valve IE, and $14.4 \%$ (20 out of 138 ) had nonvalvular IE. Among the cases involving a native valve, $25.6 \%$ (23 out of 90) had undergone previous valve repair. Across all
TABLE 2. Infective organisms isolated by blood cultures

\begin{tabular}{|c|c|}
\hline Organism & Result \\
\hline \multicolumn{2}{|l|}{ Gram positive } \\
\hline Staphylococcus spp & $47(34.1)$ \\
\hline$S$ aureus & $35(25.4)$ \\
\hline Methicillin-sensitive $S$ aureus & $29(21.0)$ \\
\hline Methicillin-resistant $S$ aureus & $6(4.3)$ \\
\hline Coagulase-negative staphylococci & $12(8.7)$ \\
\hline S epidermidis (or unspecified) & $7(5.1)$ \\
\hline$S$ capitis & $2(1.4)$ \\
\hline S lugdunensis & $2(1.4)$ \\
\hline$S$ warneri and $S$ capitis & $1(0.7)$ \\
\hline Streptococcus spp & $39(28.3)$ \\
\hline$S$ viridans & $25(18.1)$ \\
\hline Unspecified & $10(7.2)$ \\
\hline$S$ sanguis & $6(4.3)$ \\
\hline$S$ mitis & $4(2.9)$ \\
\hline$S$ mutans & $1(0.7)$ \\
\hline$S$ oralis & $4(2.9)$ \\
\hline S pneumonia & $7(5.1)$ \\
\hline Group B streptococci & $3(2.2)$ \\
\hline Others & $4(2.9)$ \\
\hline Corynebacterium diptheriae & $5(3.6)$ \\
\hline \multicolumn{2}{|l|}{ Gram negative } \\
\hline \multicolumn{2}{|l|}{ HACEK organisms } \\
\hline Haemophilus influenzae & $2(1.4)$ \\
\hline Kingella kingae & $5(3.6)$ \\
\hline Pseudomonas spp & $3(2.2)$ \\
\hline Klebsiella spp & $3(2.2)$ \\
\hline Serratia marcesens & $1(0.7)$ \\
\hline Enterococcus spp & $3(2.2)$ \\
\hline Enterobacter cloacae & $1(0.7)$ \\
\hline Streptobacillus moniliformis & $1(0.7)$ \\
\hline Escherichia coli spp & $1(0.7)$ \\
\hline Stenotrophomonas maltophilia & $1(0.7)$ \\
\hline \multicolumn{2}{|l|}{ Fungi } \\
\hline Candida parapsilosis & $2(1.4)$ \\
\hline
\end{tabular}

Values are presented as $\mathrm{n}(\%) . \quad H A C E K$, Haemophilus, Aggregatibacter (previously Actinobacillus), Cardiobacterium, Eikenella, Kingella.

patients with valvular IE, the aortic valve (39 out of 138 [28.3\%]) was the most common site of infection.

\section{Microbiology and Antibiotic Treatment}

Positive blood cultures were obtained in $79.0 \%$ of patients (109 out of 138). The identified organisms are summarized in Table 2. More than 1 organism was isolated by blood culture in $8.0 \%$ of patients (11 out of 138 ). Gram-positive infections (93 out of 109 [85.3\%]) were more common than gram-negative infections (16 out of 109 [14.7\%]). S aureus (35 out of 138 [25.3\%]), viridans group streptococci (25 out of 138 [18.1\%]), and CoNS (12 out of 138 [8.7\%]) were the 3 most commonly isolated organisms. Among patients with $S$ aureus infection, 6 were methicillin-resistant organisms. The incidence of $S$ aureus infection was significantly higher in patients who 
underwent surgery during the active phase of IE, compared with patients who underwent surgery after treatment $(31.9 \%$ vs $11.4 \% ; P=.01)$. CoNS infection occurred more frequently in patients who had previous valve replacement $(18.5 \%$ vs $6.3 \% ; P=.043)$. No significant relationship was seen between left- or right-sided IE and the organism.

Invasive intravascular procedures had been performed in $15.2 \%$ of patients ( 21 out of 138 ) within 30 days before diagnosis of IE. Five patients $(3.6 \%)$ had undergone a dental procedure within 30 days of diagnosis, and $15.9 \%$ of patients (22 out of 138) had intravascular catheters in situ at the time of diagnosis of IE. In $10.1 \%$ of cases (14 out of 138), the infection could have been related to an indwelling line.

For the entire cohort, patients received preoperative antibiotic drugs for a mean of 22.1 days (median, 15 days; IQR, 4-42 days). For patients who underwent surgery while still completing a course of intravenous antibiotics (94 out of 138 [68.1\%]), the mean length of preoperative antibiotics was 16 days (median, 9 days; IQR, 3-20 days). The majority of patients continued their initially prescribed course of antibiotics. However, in $31.2 \%$ of patients (43 out of 138 ), preoperative antibiotic regimen was altered during treatment.

\section{Preoperative Complications}

Table 3 summarizes the preoperative complications of IE in our cohort. Embolic phenomena were noted in $29.0 \%$ of patients (40 out of 138). Embolic events occurred after diagnosis of IE in $14.5 \%$ of patients (20 out of 138 ). The median time from the embolic event to the date of surgery was 6 days (IQR, 2.5-38 days). Distal embolization to soft tissues or bone was the most commonly recorded event ( 22 out of 40 [55.0\%]), followed by cerebral events (13 out of 40 [32.5\%]). Embolization to multiple locations was recorded in $6.5 \%$ of patients ( 9 out of 138 ). Neither embolic phenomena overall nor embolization to specific locations were significant predictors of mortality $(P=.743)$ or reoperation $(P=.558)$. Vegetations were seen on preoperative echocardiography in $88.4 \%$ (122 out of 138). Vegetation size (largest dimension) was recorded in $43.5 \%$ of patients $(60$ out of 138). Mean size for all measured vegetations was $15.8 \pm 9.3 \mathrm{~mm}$. On average, left-sided vegetations were smaller than right-sided vegetations (mean, $13.5 \pm 9.1 \mathrm{~mm}$ vs $16.6 \pm 9.0 \mathrm{~mm} ; P=.461$ ). To account for vegetation size relative to patient size, we calculated a vegetation size to body surface area ratio. The mean value for all patients with measured vegetations was $26.2 \pm 25.2 \mathrm{~mm} / \mathrm{m}^{2}$. These ratios were also lower in left-sided IE compared with right-sided (mean, $20.3 \pm 15.9 \mathrm{~mm} / \mathrm{m}^{2}$ vs $\left.36.3 \pm 34.1 \mathrm{~mm} / \mathrm{m}^{2} ; P=.203\right)$. In patients without embolic complications at the time of diagnosis, the median time to embolism was 3 days.
TABLE 3. Preoperative complications and echocardiography findings

\begin{tabular}{lc}
\hline \multicolumn{1}{c}{ Complication } & Result \\
\hline Leaflet perforation & $34(24.6)$ \\
\hline Perivalvular abscess & $26(18.8)$ \\
\hline Mycotic aneurysm & $11(8.0)$ \\
Intracardiac fistula & $5(3.6)$ \\
\hline Severe valvular insufficiency & $46(33.3)$ \\
Aortic & $22(15.9)$ \\
Mitral & $15(10.9)$ \\
Pulmonary & $7(5.1)$ \\
Tricuspid & $4(2.9)$ \\
Heart failure & $38(27.5)$ \\
Shock & $13(9.4)$ \\
Preoperative intensive care unit admission & $27(19.6)$ \\
Any embolic events & $40(29.0)$ \\
Cerebral & $13(9.4)$ \\
Pulmonary & $9(6.5)$ \\
Musculoskeletal & $22(15.9)$ \\
Splenic & $5(3.6)$ \\
Renal & $5(3.6)$ \\
Vegetations* & \\
Aortic valve & $36(26.1)$ \\
Mitral valve & $29(21.0)$ \\
Pulmonary valve & $14(10.1)$ \\
Tricuspid valve & $18(13.0)$ \\
Pulmonary artery & $12(8.7)$ \\
Intrificial conduit or shunt & $21(15.2)$ \\
Other intracardiac & $7(5.1)$ \\
\hline & $20(14.5)$ \\
\hline
\end{tabular}

Values are presented as $\mathrm{n}(\%) .{ }^{*}$ Identified on preoperative echocardiogram.

For the whole cohort, the median time from diagnosis to surgery was 10 days (IQR, 3-64 days). In cases of left-sided IE, the median time from diagnosis to surgery was 5 days (IQR, 2-28 days), whereas in right-sided IE this was 28 days (IQR, 6-121 days). Surgery occurred within 24 hours of diagnosis in $13.8 \%$ of patients (19 out of 138). The median time to surgery from first detection of vegetation (by echocardiography or otherwise) was 9 days (IQR, 2-58 days). In the 36 patients for whom a date or time of initial embolic event was recorded, the median time from embolism to surgery was 6 days (IQR, 2.5-38 days). Patients with cerebral embolism (13 out of $138[9.4 \%]$ ) tended to receive surgery earlier (median, 4.3 days; IQR, 1.8-13.7 days).

\section{Surgical Procedures}

The nature of the surgical procedures performed for IE are summarized in Table 4 according to the active and treated IE groups. Heart failure was the most common indication for surgery in the active group (22 out of 94 patients $[23.4 \%])$. Most patients in the treated group (22 
TABLE 4. Surgical procedures performed comparing active and treated groups

\begin{tabular}{|c|c|c|c|c|}
\hline Procedure & Total $(N=138)$ & Active $(n=94)$ & Treated $(n=44)$ & $P$ value \\
\hline \multicolumn{5}{|l|}{ Valvular procedures } \\
\hline Aortic & 39 (28.3) & $31(33)$ & $8(18.2)$ & .112 \\
\hline Repair & $16(11.6)$ & $10(10.6)$ & $6(13.6)$ & .509 \\
\hline Replacement & $23(16.7)$ & $21(22.3)$ & $2(4.5)$ & .013 \\
\hline Autograft & $12(8.7)$ & $11(11.7)$ & $1(2.3)$ & .082 \\
\hline Homograft & $8(5.8)$ & $7(7.4)$ & $1(2.3)$ & .434 \\
\hline Mechanical & $3(2.2)$ & $3(3.2)$ & $0(0)$ & $-*$ \\
\hline Mitral & $37(26.8)$ & $29(30.9)$ & $8(18.2)$ & .174 \\
\hline Repair & $31(22.5)$ & $24(25.5)$ & 7 (15.9) & .269 \\
\hline Replacement & $6(4.3)$ & $5(5.3)$ & $1(2.3)$ & .666 \\
\hline Homograft & $1(0.7)$ & $0(0)$ & $1(2.3)$ & -* \\
\hline Mechanical & $5(3.6)$ & $5(5.3)$ & $0(0)$ & .322 \\
\hline Pulmonary & $55(39.9)$ & $43(45.7)$ & $15(34.1)$ & .073 \\
\hline Repair & $17(12.3)$ & 14 (14.9) & $3(6.8)$ & .222 \\
\hline Replacement & $38(27.5)$ & $29(30.9)$ & $12(27.3)$ & .290 \\
\hline Homograft & $26(18.8)$ & $21(22.3)$ & $6(13.6)$ & .169 \\
\hline Xenograft & $12(8.7)$ & $8(8.5)$ & $6(13.6)$ & .815 \\
\hline Tricuspid repair & $24(17.4)$ & $16(17.0)$ & $8(18.2)$ & .728 \\
\hline Any replacement & $52(37.7)$ & $41(43.6)$ & $11(25.0)$ & .065 \\
\hline Any repair & $79(57.2)$ & $58(61.7)$ & $21(47.7)$ & .256 \\
\hline \multicolumn{5}{|l|}{ Concomitant/other procedures } \\
\hline Ventricular septal defect closure & $22(15.9)$ & $8(8.5)$ & $14(31.8)$ & $<.001$ \\
\hline Pulmonary artery augmentation & $15(10.9)$ & $11(11.7)$ & $4(9.1)$ & .741 \\
\hline Atrial septal defect closure & $8(5.8)$ & $7(7.4)$ & $1(2.3)$ & .257 \\
\hline Repair of aortic arch/aneurysm & $9(6.5)$ & $8(8.5)$ & $1(2.3)$ & .276 \\
\hline Right ventricular outflow tract resection & $7(5.1)$ & $4(4.3)$ & $3(6.8)$ & .434 \\
\hline Shunt takedown & $4(2.9)$ & $3(3.2)$ & $1(2.3)$ & $-*$ \\
\hline Patent ductus arteriosus ligation & $2(1.4)$ & $2(2.1)$ & $0(0)$ & -* \\
\hline Superior vena cava repair & $2(1.4)$ & $2(2.1)$ & $0(0)$ & $-*$ \\
\hline Blalock-Taussig shunt & $2(1.4)$ & $1(1.1)$ & $1(2.3)$ & $-*$ \\
\hline
\end{tabular}

Significant $P$ values are shown in bold. Values are presented as $\mathrm{n}(\%) . *$ Insufficient numbers to analyze.

out of 41 patients [53.7\%]) primarily required surgery for repair of the cardiac lesion related to their IE episode. Aortic valve replacement was significantly more common in the active group $(91.3 \%$ vs $8.7 \% ; P=.009)$. Separate or nonvalvular procedures were sometimes performed alone or concurrently with surgery for IE. The most common concomitant procedure was ventricular septal defect closure (22 out of 138 patients [15.9\%]), which includes cases of patch repair or replacement.

Over the study period, 41 patients required at least 1 reoperation. There were 7 cases $(5.1 \%)$ of early reoperation, all of which occurred within 30 days of initial surgery. Twelve patients $(8.7 \%)$ required reoperation for dysfunctional valves that were repaired or replaced at initial surgery. Reoperation was performed primarily due to somatic growth in 10 patients $(7.2 \%)$. Eleven patients $(8.0 \%)$ underwent planned reoperation for correction of pre-existing cardiac defects. There was a single case $(0.7 \%)$ of late reoperation for recurrent endocarditis 2.5 years after initial surgery.

\section{Outcomes}

Overall operative mortality was $5.8 \%$ (8 out of 138 ) for the whole cohort and occurred exclusively in patients with IE in systemic circulation. Operative mortality in children with biventricular circulation was $8.7 \%$ (6 out of 69 ) in left-sided IE and $0 \%$ in 59 children with right-sided IE. Operative mortality in children with univentricular circulation was $20 \%$ ( 2 out of 10 ); both children had IE of the atrioventricular valve. Operative mortality for aortic valve IE was $12.8 \%$ (5 out of 39 ) and $3.3 \%$ ( 1 out of 30 ) for isolated mitral valve IE in children with biventricular repair. Overall survival at 5, 15, and 25 years was 91.5\% (95\% confidence interval [CI], 85.1\%-95.2\%), 84.7\% (95\% CI, 75.4\%-90.7\%), and 79.1\% (95\% CI, 66.3\%-87.5\%), respectively (Figure $1, A$ ). When comparing active and treated subgroups, no significant differences in survival (log-rank test $P=.096$ ) or rates of reoperation (log-rank test $P=.397$ ) were noted (Figure $1, B$ ). Survival for the subgroup of patients with IE involving systemic circulation, including those with univentricular physiology, at 5, 15, and 


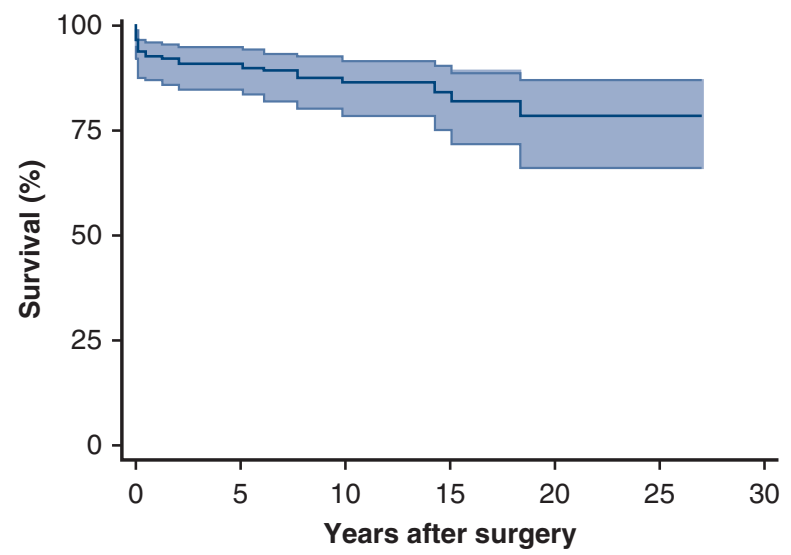

$\begin{array}{llllllll}\text { A } & \text { Number at risk } & & & & & & \\ & 138 & 92 & 61 & 39 & 14 & 6 & 0\end{array}$

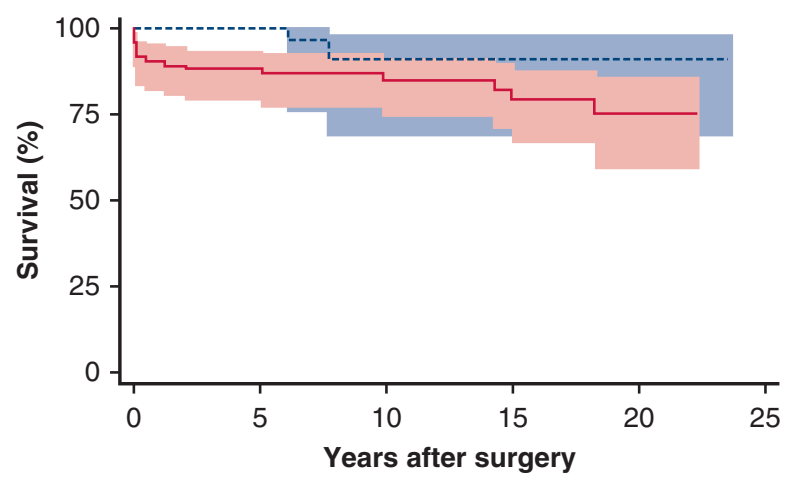

Number at risk

\begin{tabular}{|c|c|c|c|c|}
\hline Treated 4 & 28 & 17 & 9 & 4 \\
\hline Active & 64 & 44 & 30 & 10 \\
\hline
\end{tabular}

B

IE: Infective Endocarditis

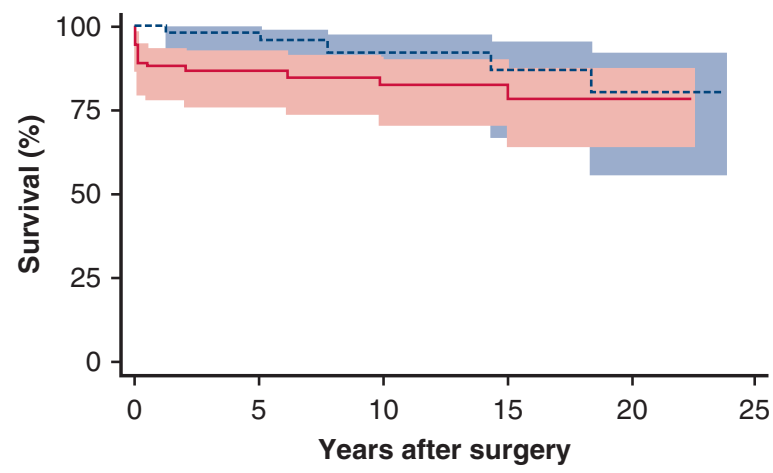

Number at risk

\begin{tabular}{rrrrrrr} 
Right-sided IE & 59 & 42 & 24 & 17 & 7 & 4 \\
Left-sided IE & 79 & 50 & 37 & 22 & 7 & 2 \\
\cline { 4 - 6 } & & ------- Right-sided IE & & \multicolumn{2}{l}{ Left-sided IE } &
\end{tabular}

FIGURE 1. Kaplan-Meier curves. A, Survival for the entire cohort. B, Comparison of active and treated groups. C, Comparison of left and right-sided infective endocarditis $(I E)$. 

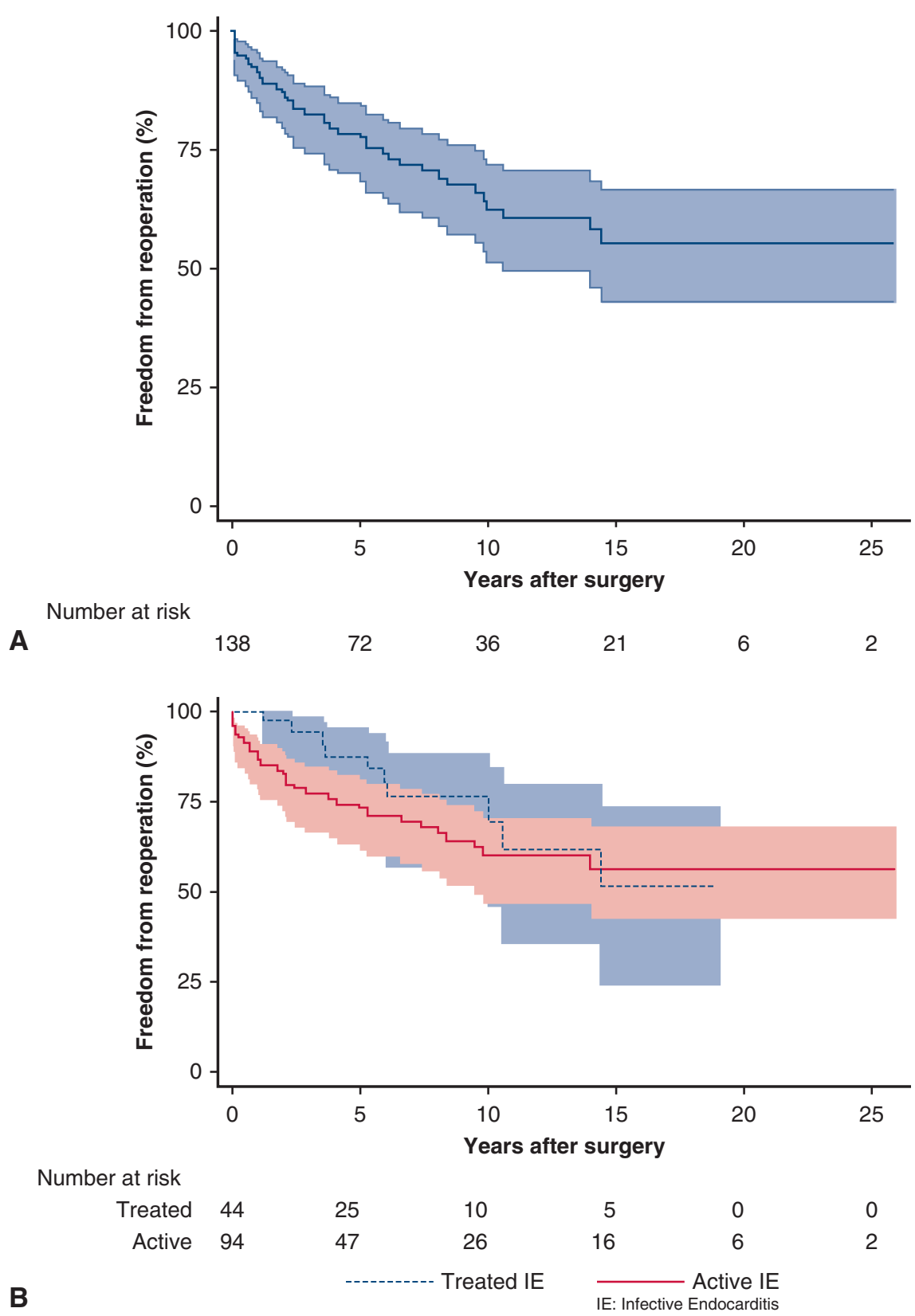

FIGURE 2. Kaplan-Meier curves. A, Freedom from all-cause reoperation for the entire cohort. B, Comparison of active and treated groups. IE, Infective endocarditis.

25 years was $86.6 \%(95 \%$ CI, 76.4\%-92.5\%), $82.5 \%$ $(95 \%$ CI, 70.8\%-89.8\%), and $78.7 \% \quad(95 \% \quad \mathrm{CI}$, $64.5 \%-87.7 \%$ ), respectively, whereas survival for those with isolated right-sided IE at the same time points was $98.1 \% \quad(95 \%$ CI, 87.1\%-99.7\%), 87.2\% (95\% CI, $67.2 \%-95.4 \%$ ), and 80.5\% (95\% CI, 55.8\%-92.2\%), respectively. Overall, late mortality occurred in 5 of 79 patients with IE in systemic circulation during the follow-up period, whereas this occurred in 5 of 59 patients with isolated right-sided IE. Long-term survival differences between left- and right-sided IE did not reach significance (log-rank test $P=.161$ ) (Figure 1, $C$ ). Freedom from all-cause reoperation at 5,15 , and 25 years was $77.3 \% \quad(95 \% \quad$ CI, 68.3\%-84.0\% $), \quad 55.5 \% \quad(95 \% \quad$ CI, $43.0 \%-66.3 \%$ ), and 55.5\% (95\% CI, 43.0\%-66.3\%), respectively (Figure 2).

On multivariable analysis (Table 5), risk factors independently associated with death were age (HR, 0.88; $P=.015)$, prosthetic valve IE (HR, 3.86; $P=.020)$, CoNS (HR, 4.52; $P=.015)$, increased duration of preoperative antibiotic therapy (HR, 1.02; $P=.009)$, shock (HR, 3.68; $P=.028$ ), and aortic valve replacement 
TABLE 5. Summary of multivariable predictors of outcome for the entire cohort

\begin{tabular}{lcc}
\hline \multicolumn{1}{c}{ Risk factor } & $\begin{array}{c}\text { Hazard ratio } \\
(\mathbf{9 5} \% \text { confidence } \\
\text { interval })\end{array}$ & $\begin{array}{c}\boldsymbol{P} \\
\text { value }\end{array}$ \\
\hline Multivariable predictor of death & & \\
$\quad$ Age & $0.88(0.80-0.98)$ & .015 \\
Prosthetic valve infective endocarditis & $3.86(1.24-12.0)$ & .020 \\
Coagulase-negative staphylococci & $4.52(1.35-15.1)$ & .015 \\
Duration of preoperative antibiotic therapy & $1.02(1.00-1.03)$ & .009 \\
Shock & $3.68(1.15-11.7)$ & .028 \\
Aortic valve replacement & $3.22(1.03-10.0)$ & .044 \\
Multivariable predictors of reoperation & & \\
$\quad$ Prior cardiac surgery & $2.00(1.04-3.85)$ & .037 \\
Perivalvular abscess & $2.83(1.44-5.59)$ & .003 \\
\hline
\end{tabular}

(HR, 3.22; $P=.044)$. Risk factors independently associated with reoperation were prior cardiac surgery (HR, 2.00; $P=.037$ ) and perivalvular abscess (HR, 2.83; $P=.003)$.

In children with left-sided IE (Table 6), risk factors independently associated with death were heart failure (HR, 18.8; $P=.025)$ and vegetation size to body surface area ratio (in $\left.\mathrm{mm} / \mathrm{m}^{2}\right)(\mathrm{HR}, 1.06 ; P=.008)$. This vegetation size ratio was also an independent predictor of mortality within 30 days (odds ratio, $1.07 ; P=.024$ ), whereas cardiogenic shock was an independent predictor of postoperative embolic events (odds ratio, 5.91; $P=.019$ ). For 38 patients with left-sided IE with recorded vegetation size (range, $5.3-76.7 \mathrm{~mm} / \mathrm{m}^{2}$; mean, $20.3 \pm 15.9 \mathrm{~mm} / \mathrm{m}^{2}$; median, $14.1 \mathrm{~mm} / \mathrm{m}^{2}$ [IQR, 9.0 to 27.5$]$ ) the operative mortality was $16 \%$ ( 6 of 38 ) and for every $1 \mathrm{~mm} / \mathrm{m}^{2}$ increase in vegetation size to the body surface area ratio, the relative risk of operative mortality increased by $7 \%$, and the absolute risk of operative mortality increased by $1.1 \%$ (odds ratio, 1.069).

For patients with right-sided IE versus left-sided IE (Table 7), length of preoperative antibiotics (HR, 1.04; $P=.001)$ and shock (HR, 18.8; $P=.033$ ) were independent predictors of death.

Freedom from recurrence of IE at 25 years was $94.7 \%$ (95\% CI, 87.7\%-97.8\%) (Figure 3).

Among patients in whom evidence of cerebral embolism was detected, $15.4 \%$ ( 2 out of 13) had residual neurologic deficits at the time of final follow-up.

\section{DISCUSSION}

Currently, recommendations for surgical management of pediatric IE are an extension of guidelines for IE management in adults. ${ }^{3}$ The decision on whether to operate to prevent primary embolic events remains problematic due to conflicting data on potential predictors of embolization. ${ }^{7,8}$

Reports on the outcome surgery for IE in children have been limited to small series of patients. ${ }^{9}$ Russell and colleagues ${ }^{10}$ described 34 IE patients who had surgery between 1990 and 2011. Overall operative mortality was $15 \%$ ( 5 out of 34 patients) but mortality for left-sided and right-sided IE was not specifically mentioned. They also reported a reoperation rate of $28 \%$ ( 10 out of 34 patients) mainly due to somatic growth. Long term outcomes were not reported. Hickey and colleagues ${ }^{11}$ reported 30 patients who had surgery for IE between 1978 and 2007. Operative mortality was $10 \%$ (3 out of 30 ) and 5-year survival and freedom from reoperation was $84 \%$ and $80 \%$, respectively. Rates of septic emboli were high in this study, occurring in 13 patients $(46 \%)$ ). Few reports in children have investigated the influence of the timing of surgery on outcomes in children. Shamszad and colleagues ${ }^{12}$ described 46 pediatric patients who underwent surgery for IE at a single center from 1996 to $2010,76 \%$ (35 out of 46) of whom underwent surgery within 7 days of diagnosis, and $54 \%$ ( 25 out of 46 ) within 3 days or fewer. Overall mortality was $2.2 \%$ with 1 death $(4 \%)$ in 25 patients with left-sided IE and no deaths in patients with right-sided IE. Estimated survival was $81 \% \pm 11 \%$ at 10 years. They demonstrated that surgery within 3 days of diagnosis was safe and did not increase the risks of reoperation or mortality. In a report from our institution in 1992, Nomura and colleagues ${ }^{13}$ highlighted the need for surgery during active or acute infection in select patients.

Overall, children who had surgery for IE at our institution had good long-term outcomes. Operative mortality in our cohort was $5.8 \%$ ( 8 out of 138). There was a gradual decline in survival over time, with $79.1 \%$ surviving at 25 years follow-up. In our patients, younger age, prosthetic valve IE, infection with CoNS, increased duration of preoperative antibiotic therapy, shock, and the need for aortic valve replacement were all independently associated with mortality in multivariable analysis.

In our study, age distribution was characterized by a peak in infancy and relatively even spread across other age

TABLE 6. Multivariable predictors of early adverse outcome for patients with left-sided infective endocarditis

\begin{tabular}{llcc}
\hline \multicolumn{1}{c}{ Outcome } & \multicolumn{1}{c}{ Risk factor } & Odds ratio $(\mathbf{9 5} \%$ confidence interval) & $\boldsymbol{P}$ value \\
\hline Early mortality (within $30 \mathrm{~d})$ & Vegetation size/body surface area ratio $\left(\mathrm{mm} / \mathrm{m}^{2}\right)$ & $1.069(1.01-1.13)$ & .024 \\
Postoperative embolic event & Shock & $5.905(1.33-26.1)$ & .019 \\
\hline
\end{tabular}


TABLE 7. Multivariable predictors of death for patients with left-sided versus right-sided infective endocarditis (IE)

\begin{tabular}{llcl}
\hline Patient group & \multicolumn{1}{c}{ Risk factor } & Hazard ratio $(\mathbf{9 5} \%$ confidence interval) & $\boldsymbol{P}$ value \\
\hline Left-sided IE & Heart failure & $18.81(1.439-245.8)$ & .025 \\
& Vegetation size/body surface area ratio & $1.064(1.017-1.114)$ & .008 \\
Right-sided IE & Length of preoperative antibiotics & $1.041(1.015-1.067)$ & .001 \\
& Shock & $18.83(1.275-278.3)$ & .033 \\
\hline
\end{tabular}

$I E$, Infective endocarditis.

groups. Russell and colleagues ${ }^{10}$ reported peaks in both infancy and adolescence. IE in younger patients has been attributed to the prolonged use of central venous catheters in infants and younger children. ${ }^{1}$ Indeed, in our cohort, $77 \%$ of catheter-associated infections (17 out of 22 ) occurred in children under 10 years. However, no significant association was noted between nosocomial IE and poor outcomes. Younger children in our study were also more likely to undergo surgery while still receiving antibiotics (odds ratio, 0.90; 95\% CI, 0.85-0.96; $P=.001$ ) suggesting elevated disease severity and awareness for the risks in this patient group.

Infection of prosthetic material or valve occurred in $21.5 \%$ of our patients ( 29 out of 138 ). Recommendations by the American Heart Association suggest valve replacement surgery in most patients with prosthetic valve infection caused by $S$ aureus. ${ }^{3}$ Our experience suggests that these patients also have poorer long-term survival. The reasons for this are likely to be multifactorial. The prosthetic material surface not only provides a surface susceptible to bacterial colonization but is also often difficult to sterilize. ${ }^{14}$ Complications such as abscess formation are said to be more common in cases of prosthetic valve IE ${ }^{15}$ although, the incidence of perivalvular abscess was similar between cases of native IE and prosthetic IE in our study.

In our patients, the incidence of CoNS infection was approximately 3 times greater in patients with prosthetic IE in comparison to native IE. This relationship is consistent with reports from other studies in adults. ${ }^{16}$ CoNS was the third most common bacterial isolate in our study, which supports recent data on microbiology trends in pediatric IE. ${ }^{17}$ CoNS infection was also identified as a predictor of death in multivariable analysis. These organisms are sometimes multiresistant and can form biofilms that confer additional protection against sterilization. ${ }^{18}$ In adults, the association of CoNS infection with greater morbidity and mortality in cases of prosthetic valve IE have been previously recognized. ${ }^{16}$

As expected, $S$ aureus was the most commonly isolated organism in our patients. Unlike in other studies, ${ }^{11,12,19}$ we did not find an association between $S$ aureus infection and adverse outcomes. Relative to other patients, a greater proportion of patients with $S$ aureus infection experienced embolic events. However, this difference did not reach statistical significance $(41.2 \%$ vs $26.0 \% ; P=.095)$. We

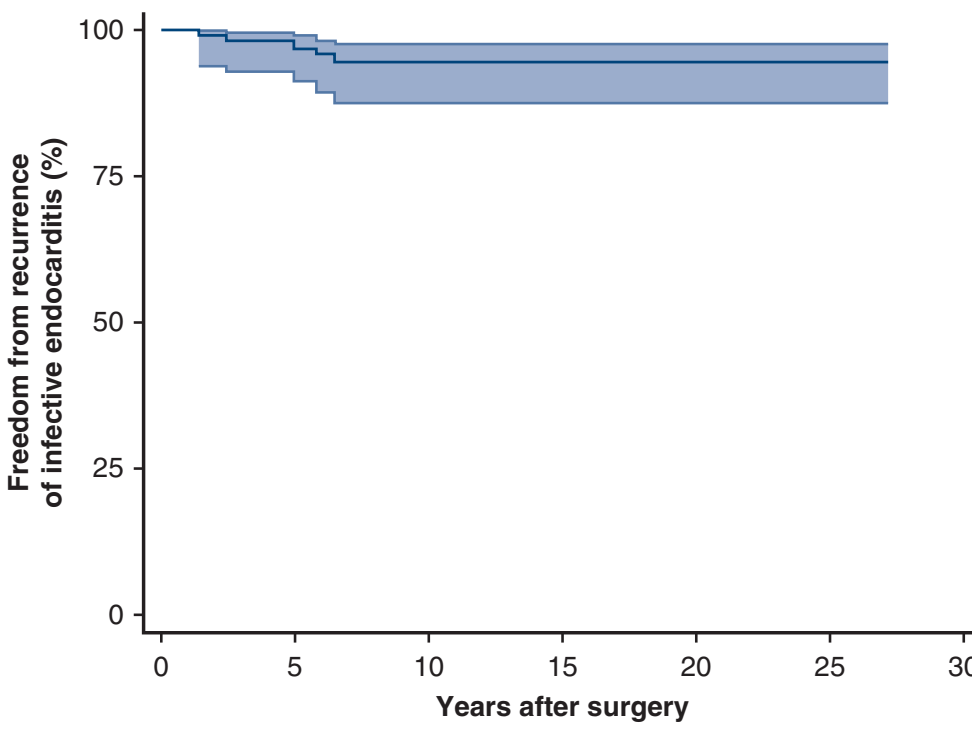

Number at risk

$\begin{array}{lllllll}138 & 90 & 57 & 36 & 12 & 5 & 0\end{array}$

FIGURE 3. Kaplan-Meier curve showing freedom from recurrence of infective endocarditis. 
noted that patients with $S$ aureus IE were more than 3 times more likely to have surgery before completing antibiotic treatment. This suggests a tendency toward more aggressive surgical management in these patients, which may mitigate the risk associated with $S$ aureus infection.

The ideal timing of surgery for IE is a controversial topic in both pediatric and adult medicine. The length of preoperative antibiotic treatment was the only measure that significantly correlated with increased mortality in our patients. Risk of death increased slightly for each additional day patients received antibiotics during the preoperative period (HR, 1.02; $P=.009$ ). Because our cohort does not include patients diagnosed with IE who were treated medically, we are unable to draw definite conclusions about the effectiveness of longer antibiotic therapy. The finding may be explained by the fact that our cohort selects for patients with right-sided IE that have failed medical therapy, and thus are more likely to have poor outcomes. We also observed that new embolic phenomena occurred at a median time of 3.2 days following diagnosis of IE. Furthermore, there was no difference in outcomes between patients who underwent surgery during the active phase of disease, and those who underwent delayed surgery. Based on these observations, it would seem reasonable to recommend surgery within 3 days of diagnosis in those patients with an indication for surgery.

Across many retrospective cohorts, vegetation size and location has been observed to influence the risk of death and adverse outcomes such as embolic phenomena. ${ }^{8,20}$ In combined cohorts of adults and children, Yoshinaga and colleagues $^{21}$ observed that vegetation size $15 \mathrm{~mm}$ or larger was a risk factor for death and remarked that it may be more useful to account for vegetation size relative to patient body size especially in infants or children. Our study adjusted vegetation sizes (in patients with recorded measurements) for body surface area, calculated from height and weight. Indeed, we found that adjusted vegetation size was a significant independent predictor of early mortality (HR, $1.069 ; P=.024)$ and overall mortality (HR, 1.064; $P=.008$ ), but only in children with left-sided IE. Because infants comprised $20 \%$ of our cohort, absolute vegetation size was not correlated with any adverse outcomes. There is a close correlation between age and body size, so these findings demonstrate the need to consider relative vegetation size, especially in cases of left-sided IE in small, young children.

IE of the aortic valve was present in $28.3 \%$ of our patients (39 out of 138 patients). According to European guidelines, heart failure due to severe aortic valve dysfunction is an indication for surgery. ${ }^{22}$ Heart failure in IE is strongly related to mortality, with the highest degree of failure associated with aortic valve destruction. ${ }^{23}$ Periannular extension of infection occurs most commonly in aortic valve endocarditis, ${ }^{24}$ a feature of more severe disease. In our cohort, aortic valve replacement was performed in $16.7 \%$ of our patients (23 out of 138) and was associated with poorer long-term survival. In contrast, none of the 16 patients who underwent aortic valve repair died during the follow-up period. Hickey and colleagues ${ }^{11}$ advocated for the avoidance of valve replacement where possible and reported good outcomes following valve repair despite advanced disease in some patients. Our findings support this approach, with our group favoring valve repair when feasible, utilizing treated autologous pericardium for native valve reconstruction where possible. ${ }^{25,26}$

Freedom from recurrence of IE occurred was $95 \%$ at 25 years follow-up, and only 2 patients in our study required reoperation for recurrent endocarditis after transition to adult care. The last recurrence of endocarditis occurred at 6.5 years of follow-up. This suggests that surgery is highly effective in curing IE in children, and recurrent episodes are very uncommon.

However, all-cause reoperation rates were high. Russell and colleagues ${ }^{10}$ reported a similar finding, where most reoperations were in children who initially underwent valve replacement and subsequently required a larger prosthesis. In our study, pre-existing heart defects and perivalvular abscess were independently associated with reoperation, whereas valve replacement on its own was not. Perivalvular abscess is said to indicate uncontrolled infection, and is most often related to the aortic valve or mitral valve. ${ }^{27}$ Annuloplasty, extensive reconstruction, or valve/root replacement are often required and are associated with higher morbidity and mortality. ${ }^{15,28}$ It is possible that the complex nature of procedures performed in these patients may necessitate earlier reoperation.

This study is limited by its retrospective nature. Due to the long follow-up period, time-related bias could potentially affect results as treatment approaches change over time. The association between adjusted vegetation size and increased mortality in left-sided IE was an important finding of our study; however, vegetation size was only recorded in $43.5 \%$ of patients. Also, the close correlation between patient age and body size was a potential confounder. Although this is the largest study on surgery in children with IE, our relatively limited sample size may have prevented further detailed analysis or demonstration of potential risk factors. The lack of data on IE patients who did not have surgery prevented more direct comparisons of approaches to therapy.

\section{CONCLUSIONS}

Children undergoing surgery for IE had good long-term survival, and recurrence of endocarditis was uncommon. However, the long-term burden of reoperation was high, with $45 \%$ of patients requiring reoperation by 25 years. Surgery during the active phase of endocarditis did not increase risk of mortality or reoperation. In children with 
left-sided IE, vegetation size indexed to body surface area was identified as a risk factor for both adverse events and death, suggesting that it may be a useful prognostic factor.

\section{Conflict of Interest Statement}

Dr d'Udekem is a consultant for Actelion and MSD. Dr Brizard serves on the advisory board of Admedus. All other authors have nothing to disclose with regard to commercial support.

\section{References}

1. Elder RW, Baltimore RS. The changing epidemiology of pediatric endocarditis. Infect Dis Clin North Am. 2015;29:513-24.

2. Day MD, Gauvreau K, Shulman S, Newburger JW. Characteristics of children hospitalized with infective endocarditis. Circulation. 2009;119:865.

3. Baltimore RS, Gewitz M, Baddour LM, Beerman LB, Jackson MA, Lockhart PB, et al. Infective endocarditis in childhood: 2015 update. Circulation. 2015;132: $1487-515$.

4. Karaci AR, Aydemir NA, Harmandar B, Sasmazel A, Saritas T, Tuncel Z, et al. Surgical treatment of infective valve endocarditis in children with congenital heart disease. J Card Surg. 2012;27:93-8.

5. Li JS, Sexton DJ, Mick N, Nettles R, Fowler VG Jr, Ryan T, et al. Proposed modifications to the Duke criteria for the diagnosis of infective endocarditis. Clin Infect Dis. 2000;30:633-8.

6. Kang D-H. Timing of surgery in infective endocarditis. Heart. 2015;101:1786.

7. Kang D-H, Kim Y-J, Kim S-H, Sun BJ, Kim D-H, Yun S-C, et al. Early surgery versus conventional treatment for infective endocarditis. N Engl J Med. 2012; 366:2466-73

8. Saxena A, Aggarwal N, Gupta P, Juneja R, Kothari SS, Math R. Predictors of embolic events in pediatric infective endocarditis. Indian Heart J. 2011;63: 237-40.

9. Murakami T, Niwa K, Yoshinaga M, Nakazawa M. Factors associated with surgery for active endocarditis in congenital heart disease. Int J Cardiol. 2012; 157:59-62.

10. Russell HM, Johnson SL, Wurlitzer KC, Backer CL. Outcomes of surgical therapy for infective endocarditis in a pediatric population: a 21 -year review. Ann Thorac Surg. 2013;96:171-4.

11. Hickey EJ, Jung G, Manlhiot C, Sakopoulos AG, Caldarone CA, Coles JG, et al. Infective endocarditis in children: native valve preservation is frequently possible despite advanced clinical disease. Eur J Cardiothorac Surg. 2009;35:130-5.

12. Shamszad P, Khan MS, Rossano JW, Fraser CD Jr. Early surgical therapy of infective endocarditis in children: a 15-year experience. J Thorac Cardiovasc Surg. 2013;146:506-11.

13. Nomura F, Penny DJ, Menahem S, Pawade A, Karl TR. Surgical intervention for infective endocarditis in infancy and childhood. Ann Thorac Surg. 1995;60:90-5.
14. Rushani D, Kaufman JS, Ionescu-Ittu R, Mackie AS, Pilote L, Therrien J, et al Infective endocarditis in children with congenital heart disease: cumulative incidence and predictors. Circulation. 2013;128:1412-9.

15. Kirali K, Sarikaya S, Ozen Y, Sacli H, Basaran E, Yerlikhan OA, et al Surgery for aortic root abscess: a 15-year experience. Tex Heart Inst J. 2016;43:20-8

16. Lalani T, Kanafani ZA, Chu VH, Moore L, Corey GR, Pappas P, et al. Prosthetic valve endocarditis due to coagulase-negative staphylococci: findings from the International Collaboration on Endocarditis Merged Database. Eur J Clin Microbiol Infect Dis. 2006;25:365-8.

17. Tseng WC, Chiu SN, Shao PL, Wang JK, Chen CA, Lin MT, et al. Changing spectrum of infective endocarditis in children: a 30 years experiences from a tertiary care center in Taiwan. Pediatr Infect Dis J. 2014;33:467-71.

18. Takano T, Ohtsu Y, Terasaki T, Wada Y, Amano J. Prosthetic valve endocarditis caused by Staphylococcus capitis: report of 4 cases. J Cardiothorac Surg. 2011;6:131.

19. Tolan RW Jr, Kleiman MB, Frank M, King H, Brown JW. Operative intervention in active endocarditis in children: report of a series of cases and review. Clin Infect Dis. 1992;14:852-62.

20. Gonzalez-Melchor L, Kimura-Hayama E, Diaz-Zamudio M, Higuera-Calleja J, Choque C, Soto-Nieto GI. In-hospital mortality risk factors for patients with cerebral vascular events in infectious endocarditis. A correlative study of clinical, echocardiographic, microbiologic and neuroimaging findings. Arch Cardiol Mex. 2015;85:195-200.

21. Yoshinaga M, Niwa K, Niwa A, Ishiwada N, Takahashi H, Echigo S, et al. Risk factors for in-hospital mortality during infective endocarditis in patients with congenital heart disease. Am J Cardiol. 2008;101:114-8.

22. Horstkotte D, Follath F, Gutschik E, Lengyel M, Oto A, Pavie A, et al. Guidelines on prevention, diagnosis and treatment of infective endocarditis executive summary; the Task Force on Infective Endocarditis of the European society of cardiology. Eur Heart J. 2004;25:267-76.

23. Brink J, d'Udekem Y. So, vegetation size in right-sided endocarditis does no matter, or does it? Heart Lung Circ. 2016;25:419-20.

24. Ferrieri P, Gewitz MH, Gerber MA, Newburger JW, Dajani AS, Shulman ST, et al. Unique features of infective endocarditis in childhood. Circulation. 2002; 105:2115-27.

25. Delmo Walter EM, Musci M, Nagdyman N, Hubler M, Berger F, Hetzer R. Mitral valve repair for infective endocarditis in children. Ann Thorac Surg. 2007;84: 2059-65.

26. Hostasa J, Chaloupecky V, Tomek V, Tlaskal T, Janousek J. Surgical treatmen of infective endocarditis in children and young adults. Cardiol Young. 2014;24: S58.

27. Middlemost S, Wisenbaugh T, Meyerowitz C, Teeger S, Essop R, Skoularigis J et al. A case for early surgery in native left-sided endocarditis complicated by heart failure: results in 203 patients. J Am Coll Cardiol. 1991;18:663-7.

28. d'Udekem Y, David TE, Feindel CM, Armstrong S, Sun Z. Long-term results of operation for paravalvular abscess. Ann Thorac Surg. 1996;62:48-53.

Key Words: infective endocarditis, surgery, pediatric, outcomes, mortality, reoperation, timing, valve replacement 